Lewin, R. A. (1956). J. gen. Microbiol. 15, 170-185

\title{
Control of Sexual Activity in Chlamydomonas by Light
}

\author{
By R. A. LEWIN \\ Maritime Regional Laboratory, National Research Council, Halifax, \\ Nova Scotia, Canada
}

SUMMARY: Chlamydomonas moerousii is a unicellular, haploid, heterothallic alga. Suspensions containing a high proportion of active gametes are readily obtainable. The species is obligately photo-autotrophic. Organisms grown in light lose sexual activity when incubated in darkness, and only regain it when illuminated. Organisms of both mating-types require light for sexual activation. This reaction appears independent of the presence or absence of $\mathrm{CO}_{2}$. It was not found possible to replace the effect of light by the addition of any chemical or natural extract to the medium. By the use of a simple device, changes in the sexual activity of a given suspension were followed experimentally. The sensitivity of organisms to light remained approximately constant during an experimental run of 1-2 hr. Mating activity of organisms transferred to darkness remained constant for a few minutes ('dark lag') before decreasing. The length of the dark lag was a function of the duration of previous illumination. The dark lag of plus organisms was shorter than that of minus organisms under comparable conditions. After the lag period, decline of activity in darkness was approximately exponential. Rates of loss of activity between $10^{\circ}$ and $35^{\circ}$ were measured, and half-life values calculated, for each mating-type. The rate of loss of activity was approximately the same for both mating-types. Loss of sexual activity was somewhat accelerated by anaerobiosis. Resumption of sexual activity on re-illumination followed a short period of persistent inactivity ('light lag'). The length of the light lag was not dependent on the duration of the preceding dark period. At $\mathbf{2 5}^{\circ}$, the lower threshold of white light was 10 f.c. for minus organisms, 50 f.c. for plus organisms. At 300 f.c., minus organisms were reactivated between $12 \cdot 5^{\circ}$ and $35^{\circ}$, plus organisms between $17 \cdot 5^{\circ}$ and $30^{\circ}$. The action spectrum of photoactivation exhibited two peaks, around 450 and $680 \mathrm{~m} \mu$., thereby resembling the absorption spectrum of the chloroplast. Phenylurethan $\left(6 \times 10^{-4} \mathrm{M}\right)$ reversibly inhibited photosynthesis and photoactivation of sexuality, while respiration and the mating process itself were affected to a much lesser degree. It is postulated that mating activity is controlled by an intracellular hormone, activated at the plastid during illumination, and operative at the flagella.

Light has long been known to play an important role in the sexual activity of plants. In the case of Chlamydomonas and related algae, experimental observations on the action of light have been made by Klebs, Moewus, Smith and others: their work has been briefly reviewed elsewhere (Lewin, 1954). The following experiments were carried out solely with the species Chlamydomonas moereusii Gerloff, which lends itself particularly well to studies of sexual activity for the following reasons: (1) Methods have already been evolved (Lewin, 1953b) for regularly eliciting the abundant production of sexually active organisms. (2) The species is heterothallic, so that mating can be initiated at a given time by mixing suspensions of organisms of complementary mating-types. (3) Since paired organisms remain in copulo, without further fusion, for some hours, the number of pairs provides a convenient 


\section{Light and sexuality in Chlamydomonas sp.}

measure of the activity of gamete suspensions. (4) The loss of sexual activity in darkness, and reactivation of gametes by light, are processes which take place within a matter of minutes, so that many experimental investigations can be carried out without the added complications of cell ageing or cell division.

The following experiments were designed to obtain more precise quantitative information on the dynamics of these processes, and thereby to find some indications as to the chemical reactions underlying sexual activity.

\section{METHODS}

The strains of Chlamydomonas moewusii used in these investigations were originally isolated by L. Provasoli in 1948. Cultures of opposite mating-type, designated as plus and minus respectively, were maintained separately on agar slopes, growth in each being exclusively vegetative. Since sexual activity appears to be confined to young flagellated organisms recently liberated after vegetative reproduction, the following empirical method was evolved for the production of active gamete suspensions. The basal mineral medium employed (MM) contained (g./l.): $\mathrm{K}_{2} \mathrm{HPO}_{4}, 0 \cdot 2 ; \mathrm{MgSO}_{4} .7 \mathrm{H}_{2} \mathrm{O}, 0 \cdot 2 ; \mathrm{Ca}\left(\mathrm{NO}_{3}\right)_{2} .4 \mathrm{H}_{2} \mathrm{O}, 1 \cdot 0$, supplemented with $\mathrm{Fe}$ and other trace elements (Lewin, 1953b). For growth of gametes (MAC), MM medium was supplemented with (g./l.): $\mathrm{Na}$ acetate $\mathbf{2 H}_{\mathbf{2}} \mathrm{O}$, 1.0; $\mathrm{Na}_{3}$ citrate $2 \mathrm{H}_{2} \mathrm{O}, \mathbf{1} \cdot \mathbf{0}$; agar (Difco), 10.0. (1) Erlenmeyer flasks (125 ml.) containing $30 \mathrm{ml}$. medium MAC, and covered by an inverted $60 \mathrm{ml}$. beaker, were sterilized by autoclaving. After cooling, the agar surface was inoculated by pouring on a dense culture of organisms grown in mineral medium MM, and then decanting the free liquid. Two flasks were prepared in this way, one for each mating-type. The cultures were illuminated at $c .350$ f.c. under 'white' fluorescent tubes for 7 days. (2) They were then transferred to darkness and incubated for $18 \mathrm{hr}$., during which time division of each enlarged vegetative organism gave rise to 4-8 young organisms with gametic activity. (3) Such surface cultures were then flooded with $15 \mathrm{ml}$. of a weak buffer solution FM (g./l.: $\mathrm{Na}_{2} \mathrm{HPO}_{4} \cdot 7 \mathrm{H}_{2} \mathrm{O}, 0.2 ; \mathrm{CaCl}_{2} \cdot 2 \mathrm{H}_{2} \mathrm{O}, 0.05 ; \mathrm{pH} 8 \cdot 0$ ), and incubated for $1 \mathrm{hr}$. in darkness. Under these conditions abundant flagellated and motile organisms were released into the medium (Lewin, 1953 $a$ ), producing a suspension containing 5 to $10 \times 10^{6} \mathrm{organisms} / \mathrm{ml}$. (4) Suspensions prepared in this way were sexually inactive. For experiments on the loss of activity in darkness (p. 174), it was first necessary to activate the organisms by pre-illumination. In such cases the suspension to be tested was decanted into a clean flask and illuminated under the fluorescent lights for 30-120 min.

In order to follow the behaviour of plus and minus organisms separately, the following procedure was adopted. Briefly, it consisted of subjecting organisms of one mating-type to various conditions of light and temperature in a simple reaction chamber (Fig. 1 and $\mathrm{Pl}$. 1), and at intervals removing samples in which mating activity was tested against a standard suspension of gametes of the opposite type.

Uniform mating suspensions of plus and minus organisms were used for each experiment. Samples $(0.3 \mathrm{ml}$.) of organisms of one mating-type were pipetted 
into a series of small tubes $\mathbf{7 5} \times \mathbf{8} \mathbf{~ m m}$. internal diameter in a Perspex holder, and were maintained in a fully light-activated state by constant illumination under fluorescent tubes. Such suspensions will be referred to as 'tester' stocks. Organisms of the complementary mating-type to be tested were drawn by suction into the reaction chamber $F$ (see Fig. 1) through opening $D$, and were kept stirred by a slow stream of air or other gas, tap $C$ being open. At intervals the tap was closed temporarily, and a small sample ( 2 drops, c. $0 \cdot 1 \mathrm{ml}$.) expelled from $D$, by the use of a syringe connected at $A$, into one of the tubes of tester stock $G$. Pairing was generally completed in 10-15 min. (see Fig. 2) and the pairs remained motile without fusing for an indefinite period when light was excluded. The mixed suspensions were therefore left for an hour or more in



Fig. 1. Diagram of reaction chamber. $A$, to syringe; $B$, to aspirator; $C$, tap; $D$, air intake or delivery outlet for suspension; $E$, standard-taper ground joint; $F$, suspension of organisms subjected to various temperature and illumination treatments; $G$, tube with tester organisms of opposite mating-type.

darkness, and the organisms were then killed by the addition of a drop of $1 \%$ aqueous iodine. Mating activity was determined by counting the number of copulating pairs in a given volume of liquid in a haemacytometer.

For the determination of an action spectrum (p. 179), Bausch \& Lomb interference filters were used to screen light from an incandescent tungsten filament in a small projection lantern. The transmission of such filters, as measured in a Beckman spectrophotometer, is restricted to a narrow band, the intensity falling to about $10 \%$ at $20 \mathrm{~m} \mu$. on either side of the peak. The distance between the organism suspension and the light source was adjusted for each filter so that the incident light energy, as measured by a thermocouple and mirror galvanometer, was of the same value (c. $\left.500 \mathrm{ergs} / \mathrm{cm}^{2} / \mathrm{sec}\right)$.

Cultures were grown and maintained at $22^{\circ} \pm 1^{\circ}$, except in the reaction chamber, which was immersed in a water-bath thermostatically controlled at various temperatures with a fluctuation range of less than $\pm 0 \cdot 1^{\circ}$. 


\section{RESULTS}

Organism pairs as a measure of gamete activity

It may be postulated that, in the presence of an excess of active minus gametes, every plus gamete can ultimately pair if its sexual activity is retained long enough for it to find a partner. Under such conditions, the number of pairs formed will thus be a measure of the activity of gametes in the original plus suspension. Mutatis mutandis, the same would be expected for minus gametes, which are indistinguishable in size and activity. An indication of the proportionality between the numbers of active gametes and of pairs of organisms

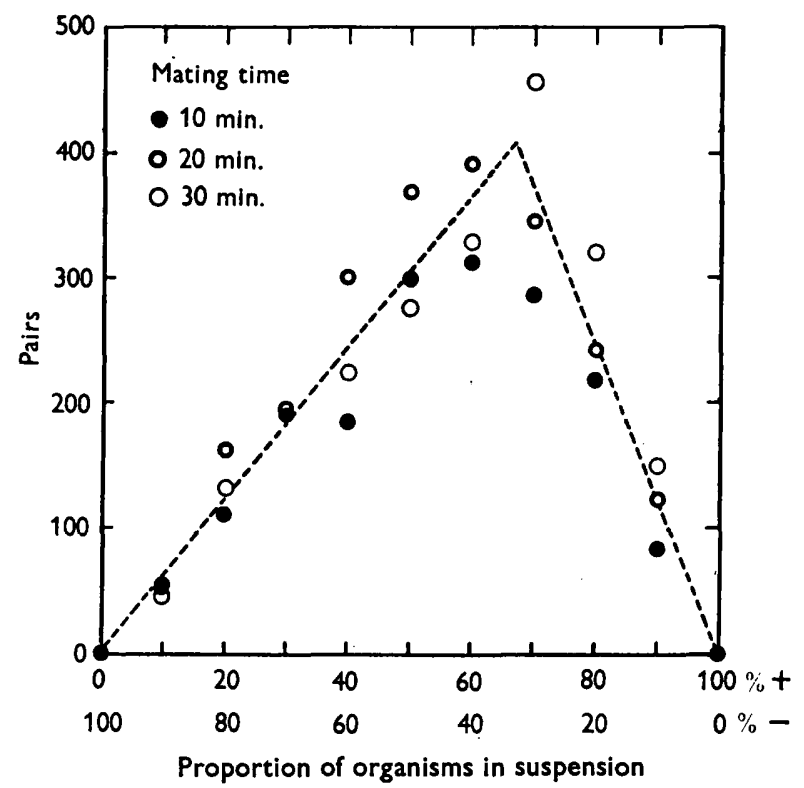

Fig. 2. Effect of relative proportions of gametes on numbers of pairs/ $\mu \mathrm{l}$. Plus suspension, 1600 organisms $/ \mu \mathrm{l}$. Minus suspension, 1800 organisms $/ \mu \mathrm{l}$. Organisms killed 10, 20, 30 min. after mixing.

was obtained from the following experiment. Samples from illuminated plus and minus suspensions containing approximately equal numbers of organisms were mixed in various proportions in light. After 10, 20 and $30 \mathrm{~min}$., samples were removed, and the organisms killed with iodine. Counts of pairs of organisms indicated that when active plus gametes were in a minority, the number of pairs was proportional to the number of plus gametes: while when the latter were in excess, the number of pairs was proportional to the minus gametes. This is shown in Fig. 2, in which two straight lines indicating direct proportionality intersect at a plus:minus ratio of organisms where there are presumably equal numbers of active gametes in the mixture. The fact that in this experiment the maximum number of pairs occurred in a mixture containing $70 \%$ plus and $30 \%$ minus organisms indicated that, in the suspension of 
plus organisms used, there happened to be a lower density of active gametes than in the minus suspension. It also appeared from this figure that mating was virtually completed within $10 \mathrm{~min}$. of mixing the suspensions.

\section{Loss of sexual activity in darkness}

The effects of illumination and darkness on a gamete suspension in the reaction chamber are shown in Fig. 3, which represents the results of a typical experiment. Similar curves were obtained when either plus or minus organisms were studied, indicating that for mating to take place both mating-types require illumination, and that both types lose activity in darkness. Since, with certain reservations, mating activity appeared to be lost at an exponential rate, pair numbers were expressed in a semi-logarithmic plot against time in darkness to give approximately a straight line. The slope of this line provided a measure of the rate of decay of mating activity under the conditions investigated. Fig. 4 illustrates the results of two experiments plotted in this way.



Fig. 3. Effect of darkness and light on sexual activity. Minus organisms tested. Pre-illumination 30 min., 300 f.c. Temperature $23^{\circ}$. $P=$ pairs $/ 0 \cdot 3 \mu l$.

In organisms which had been transferred to darkness after pre-illumination, the decay rate of mating activity was found to be dependent on the temperature at which they were maintained in the dark. The results of an extended series of experiments carried out at various temperatures between $10^{\circ}$ and $35^{\circ}$ are summarized in Table 1 , in which the slopes have been expressed as halflife values. It was not practicable to investigate temperatures below $10^{\circ}$, since such experiments would last for several hours, during which time multiplication and other changes in the illuminated suspensions (see p. 178) would tend to change the sexual activity within the suspensions to an appreciable extent.

The rate of decay was unaffected by turbulence, but was found to be somewhat accelerated in the absence of oxygen (Fig. 5). The passage of a stream of $\mathrm{CO}_{2}$-free air did not appreciably affect the course of the reaction (Fig. 6). 


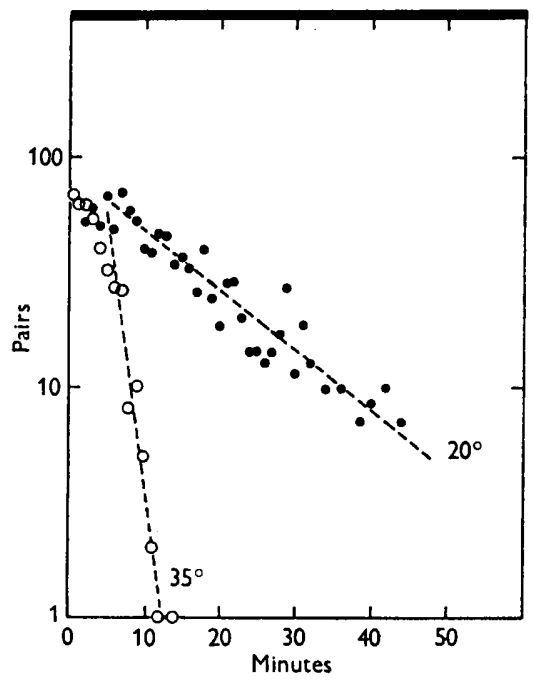

Fig. 4.

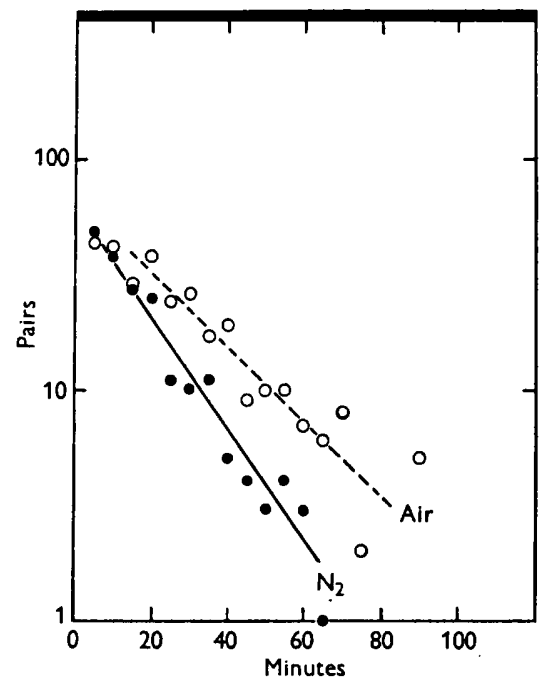

Fig. 5.

Fig. 4. Effect of temperature on loss of sexual activity in darkness. Plus organisms tested. Pre-illumination $60 \mathrm{~min} ., 300$ f.c. Pairs $/ 0.5 \mu \mathrm{l}$.

Fig. 5. Effect of oxygen on loss of sexual activity in darkness. Plus organisms tested. Pre-illumination 30 min., 300 f.c. Temperature $23^{\circ}$. Pairs $/ 0 \cdot 3 \mu l$.

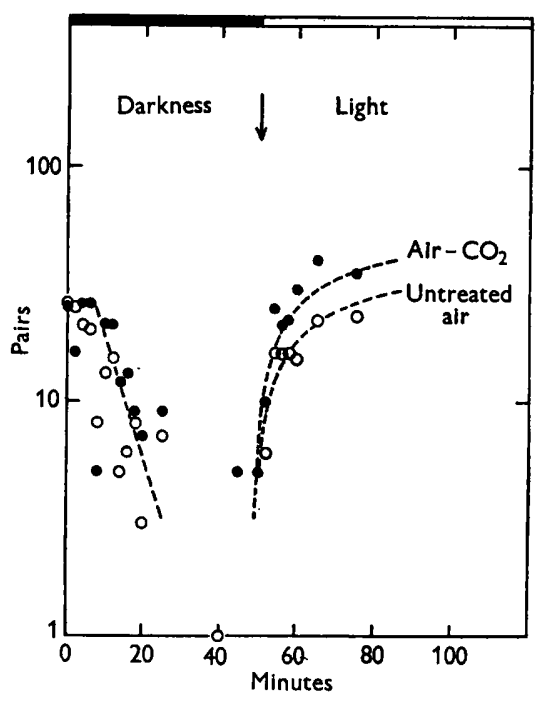

Fig. 6.

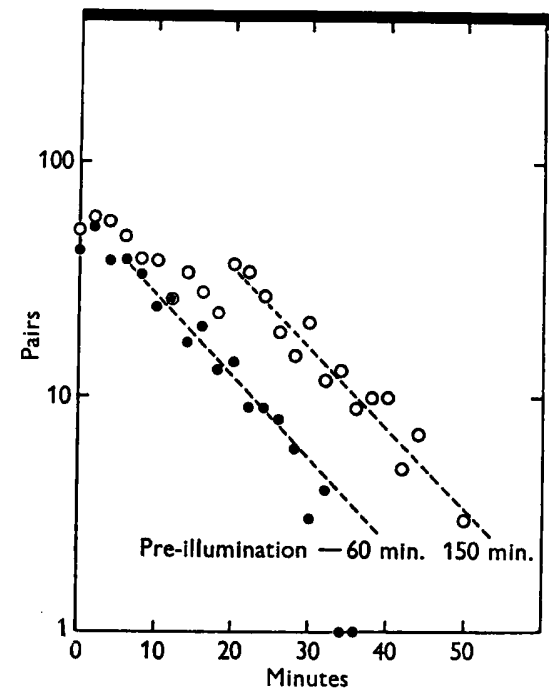

Fig. 7.

Fig. 6. Effect of presence of $\mathrm{CO}_{2}$ on activation of sexuality by light. Minus organisms tested. Pre-illumination 30 min., 300 f.c. Temperature $24^{\circ}$. Pairs $/ 0 \cdot 3 \mu l$.

Fig. 7. Effect of pre-illumination period on loss of sexual activity in darkness. Minus organisms tested. Pre-illumination 300 f.c. Temperature $23^{\circ}$. Pairs $/ 0 \cdot 3 \mu l$. 
Organisms which received only a short period of pre-illumination began to lose sexual activity as soon as they were transferred to darkness; whereas organisms illuminated for a longer period retained full mating activity in darkness for several minutes before the numbers of pairs began to fall off (Fig. 7). The lengths of the lag period observed after various durations of pre-illumination at $\mathbf{2 2 0}$ f.c. are given in Table $\mathbf{2}$. The significance of this lag period is discussed below (p. 183).

Table 1. Loss of mating activity in darkness: half-life values

Temperature $\left({ }^{\circ} \mathrm{C}.\right)$

\begin{tabular}{|c|c|c|c|c|c|c|c|}
\hline \multirow{3}{*}{$\begin{array}{c}\text { Strain } \\
\text { Plus }\end{array}$} & \multirow{3}{*}{$\begin{array}{c}\begin{array}{c}\text { Pre-illumination } \\
\text { (min.) }\end{array} \\
\mathbf{3 0}\end{array}$} & 10 & 15 & 20 & $\mathbf{2 5}$ & 30 & 35 \\
\hline & & \multicolumn{6}{|c|}{ Half-life values (min.) } \\
\hline & & 33 & 14 & 8 & $\mathbf{5}$ & 3 & $1 \cdot 33$ \\
\hline & 60 & 32 & 19 & $6 \cdot 5$ & 6 & 3 & $1 \cdot 33$ \\
\hline & 90 & 48 & $17 \cdot 5$ & $6 \cdot 5$ & $\mathbf{5 \cdot 5}$ & $3 \cdot 75$ & $\mathbf{1} \cdot \mathbf{3 3}$ \\
\hline & 120 & 36 & 18 & 7 & 6 & 4 & $1 \cdot 5$ \\
\hline Minus & 30 & $\mathbf{5 2}$ & $\mathbf{3 3}$ & 10 & 8 & $2 \cdot 75$ & $\mathbf{1 \cdot 5}$ \\
\hline & 60 & 45 & 32 & 15 & $\mathbf{9}$ & $3 \cdot 75$ & $\mathbf{1 . 5}$ \\
\hline & 90 & 49 & $\mathbf{3 3}$ & 12 & 19 & 3 & $1 \cdot 75$ \\
\hline & 120 & 35 & 28 & 11 & 7 & $4 \cdot 5$ & $\mathbf{1} \cdot \mathbf{7 5}$ \\
\hline
\end{tabular}

Table 2. Loss of mating activity in darkness: duration of lag

\begin{tabular}{|c|c|c|c|c|c|c|c|}
\hline & \multirow{2}{*}{$\begin{array}{l}\text { Pre-illumination } \\
\text { (min.) }\end{array}$} & \multicolumn{6}{|c|}{ Temperature $\left({ }^{\circ} \mathrm{C}.\right)$} \\
\hline & & \multicolumn{6}{|c|}{ Duration of lag (min.) } \\
\hline Plus & $\begin{array}{r}30 \\
60 \\
90 \\
120\end{array}$ & $\begin{array}{l}45 \\
65 \\
70 \\
72\end{array}$ & $\begin{array}{l}10 \\
19 \\
25 \\
30\end{array}$ & $\begin{array}{r}5 \\
9 \\
10 \\
19\end{array}$ & $\begin{array}{r}4 \\
6 \\
7 \\
10\end{array}$ & $\begin{array}{r}4 \\
11 \\
8 \\
10\end{array}$ & $\begin{array}{l}\mathbf{2} \\
\mathbf{5} \\
\mathbf{3} \\
\mathbf{4}\end{array}$ \\
\hline Minus & $\begin{array}{r}30 \\
60 \\
90 \\
120\end{array}$ & $\begin{array}{l}180 \\
180 \\
204 \\
175\end{array}$ & $\begin{array}{r}50 \\
145 \\
125 \\
100\end{array}$ & $\begin{array}{l}\mathbf{1 9} \\
\mathbf{2 0} \\
\mathbf{3 7} \\
\mathbf{4 8}\end{array}$ & $\begin{array}{l}10 \\
25 \\
42 \\
43\end{array}$ & $\begin{array}{r}3 \\
15 \\
22 \\
29\end{array}$ & $\begin{array}{l}2 \\
3 \\
6 \\
5\end{array}$ \\
\hline
\end{tabular}

\section{Restoration of sexual activity by light}

At intensities of white light around 200-300 f.c., reactivation of gamete suspensions was rapid, usually being almost completed in 15-20 min.; at lower intensities, pairing activity increased more slowly (Fig. 8). At $25^{\circ}$ the lower threshold for light reactivation was of the order of 10 f.c. for minus organisms, and 50 f.c. for plus organisms. With the illumination constant at 300 f.c., minus organisms were reactivated in the temperature range $12.5^{\circ}-35^{\circ}$ (Fig. 9): in the case of plus organisms the range was more restricted, $17 \cdot 5^{\circ}-30^{\circ}$ (Fig. 10).

The presence of $\mathrm{CO}_{2}$ appeared to be unnecessary for the process of sexual activation by light. Parallel samples from a uniform gamete suspension were incubated for $1 \mathrm{hr}$. in darkness, one being aerated with normal air, and the other with air from which the $\mathrm{CO}_{2}$ had been scrubbed by passage through two 


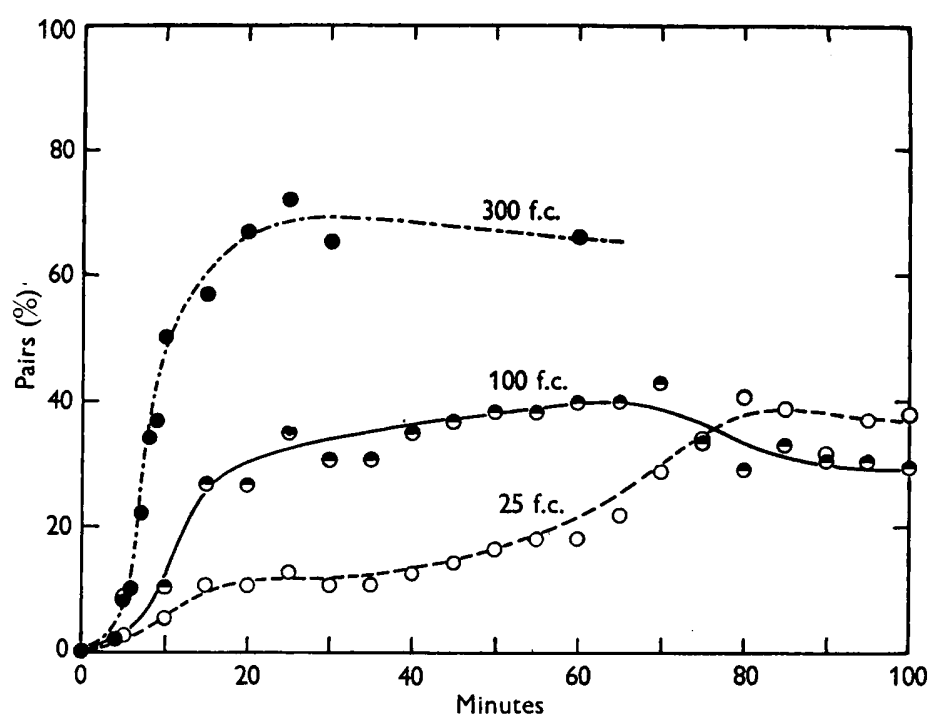

Fig. 8. Effect of light intensity on activation of sexuality. Minus organisms tested. Illumination 25, 100, 300 f.c. respectively. Temperature $25^{\circ}$. Pairs expressed as percentage of 'maximum' obtained in comparable gamete mixtures illuminated at 300 f.c. for $2 \mathrm{hr}$. Max. numbers, respectively, 182, 254, 177 pairs $/ 0.5 \mu l$.

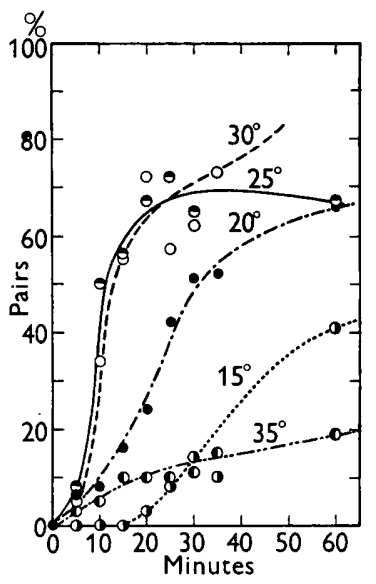

Fig. 9.

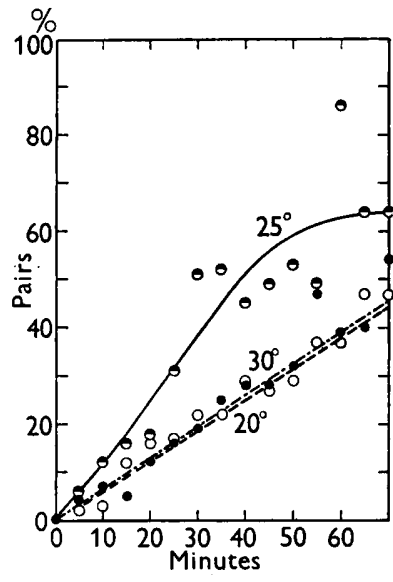

Fig. 10.

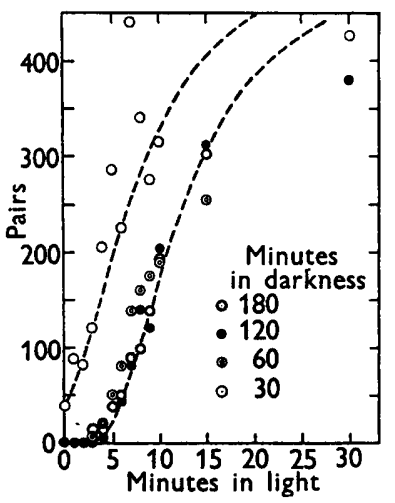

Fig. 11.

Fig. 9. Effect of temperature on activation of sexuality of minus organisms by light. Illumination 300 f.c. Temperatures $15^{\circ}, 20^{\circ}, 2^{\circ}, 30^{\circ}, 35^{\circ}$, respectively. Pairs expressed as percentage of 'maximum': see Fig. 8. Max. numbers, respectively, 79, 246, 177, 110 , 182 pairs $/ 0 \cdot 5 \mu 1$.

Fig. 10. Effect of temperature on activation of sexuality of plus organisms by light. Illumination 300 f.c. Temperatures $20^{\circ}, 25^{\circ}, 30^{\circ}$ respectively. Pairs expressed as percentage of 'maximum': see Fig. 8. Max. numbers respectively $57,51,87$ pairs $/ 0.5 \mu l$.

Fig. 11. Effect of duration in darkness on subsequent reactivation of sexuality by light. Illumination 300 f.c. Temperature $24^{\circ}$. Pairs $/ 0 \cdot 2 \mu \mathrm{l}$. 
flasks containing $10 \%(\mathrm{w} / \mathrm{v})$ sodium hydroxide. They were then illuminated without altering the aeration system, and samples were removed at intervals and tested for sexual activity. No significant difference was observed between the two activation curves, either in the rate of activation or in the level of maximum activity (Fig. 5).

Organisms in which sexual activity had been diminished, but not eliminated, by a short period in darkness showed an immediate increase in activity on illumination. Once sexual activity had been completely lost, the reactivation of gametes by light followed a short lag period, which was of similar duration in organisms subjected to as little as $\mathbf{3 0} \mathrm{min}$. of darkness, or as much as $180 \mathrm{~min}$. (Fig. 11).

\section{Variation in sensitivity to light}

In spite of precautions to obtain as uniform a suspension of gametes as possible (see p. 171), there doubtless remained some variation in the sensitivity of individual organisms to stimulation by light. In addition, it was necessary to determine to what extent experimental results might be influenced by fluctuations of overall activity in suspensions maintained in darkness under turbulent conditions in the reaction chamber. Experiments of the following type were therefore carried out. Organisms were introduced into the chamber

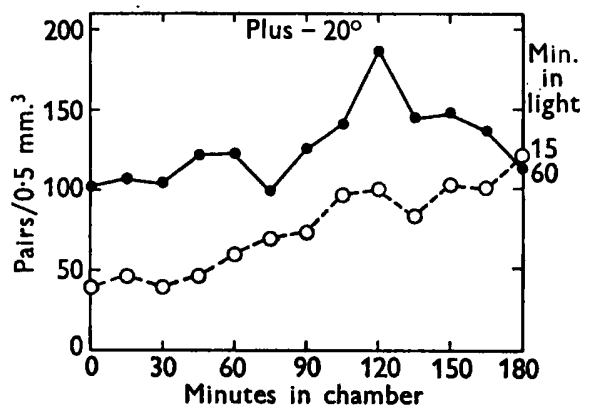

Fig. 12.

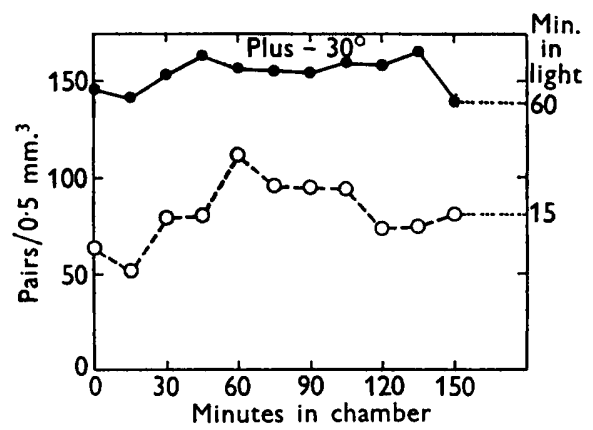

Fig. 14.



Fig. 13.

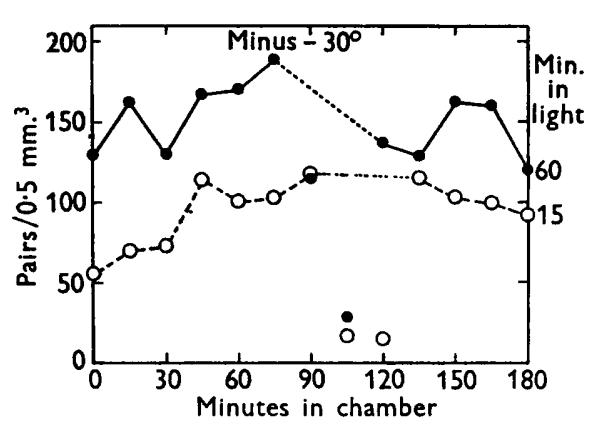

Fig. 15.

Figs. 12-15. Effect of experimental conditions on sexual response to a constant dose of light. Illumination 300 f.c.: duration 15 or $60 \mathrm{~min}$. Fig. 12, plus organisms tested. Temperature $20^{\circ}$ Fig. 13, minus organisms tested. Temperature $20^{\circ}$. Fig. 14, plus organisms tested. Temperature $30^{\circ}$. Fig. 15, minus organisms tested. Temperature $30^{\circ}$. 


\section{Light and sexuality in Chlamydomonas $s p$.}

and maintained in darkness. At $15 \mathrm{~min}$. intervals, samples were removed, mixed with tester stock (fully light-activated organisms of the complementary mating-type), and then subjected to a standard dose of illumination (15 or 60 min. at 300 f.c.). The mixtures were then transferred to darkness for the completion of the mating reaction. The results of four such experiments, carried out on each mating type at $20^{\circ}$ and $30^{\circ}$ respectively, are shown in Figs. 12-15. They indicated that over a period of hours there was some fluctuation in the overall sensitivity of gamete suspensions to light.

The causes underlying these variations of response may be complex. Organisms of both mating-types were ageing, those of the tester stock in light, the others in a turbulent aerated suspension in darkness. Not only did the number of potential gametes change with time, but apparently also the threshold value of light required for sexual activation. This emerged from the observation that the proportion of gametes capable of sexual activation was higher in suspensions receiving $1 \mathrm{hr}$. illumination than in those illuminated for only $15 \mathrm{~min}$.

Such changes of gametic sensitivity to light certainly occurred in all experiments. However, it may be noted that the drift in potential sexual activity was a gradual one, evident over a period of some hours, whereas the experiments described in the sections on 'variation in sensitivity to light' and 'action spectrum' occupied considerably shorter periods. Considering the magnitude of variation between replicate runs, the error due to drift of intrinsic sexuality can probably be ignored at this stage.

\section{Action spectrum}

A $5 \mathrm{~min}$. period of dim illumination was insufficient for complete reactivation of gametes (Fig. 8), and under such circumstances sexual activity became a function of light intensity. Samples of a uniform mixed suspension containing approximately equal numbers of dark-inactivated plus and minus

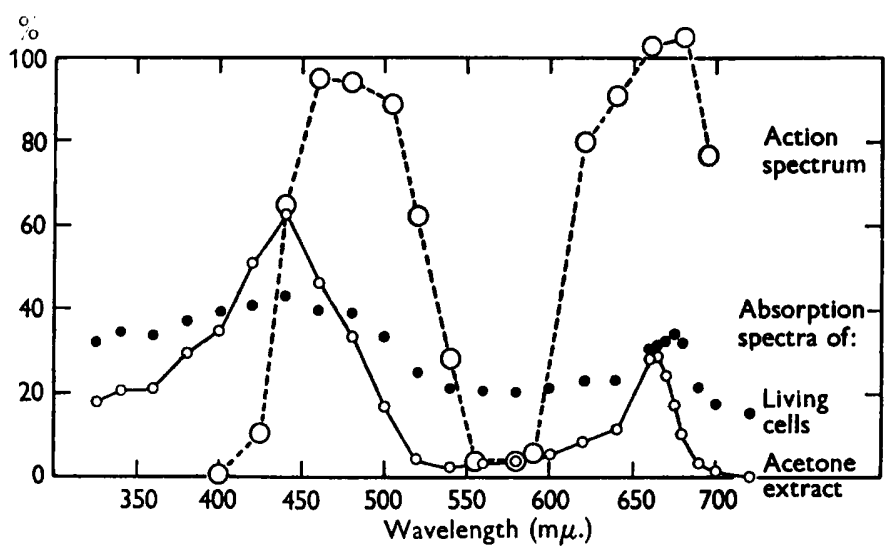

Fig. 16. Action spectrum of sexual activation by light. Plus and minus organisms mixed after $3 \mathrm{hr}$. in darkness. Temperature $20^{\circ}$. Pairs expressed as percentage of 'maximum' obtained in sample illuminated for $5 \mathrm{~min}$. in white light, then incubated in darkness. Max. 146 pairs $/ 0 \cdot 2 \mu$ l. Light absorptions determined in Beckman spectrophotometer: 'absorption' of living organisms not compensated for scattered light. 
organisms were irradiated for $5 \mathrm{~min}$. with light transmitted through interference filters, and then kept in darkness until after pairing ceased. By comparing the effectiveness of various wavelengths of light in inducing pairing, it was possible to obtain an action spectrum of sexual activation. The form of the curve, with peaks around 450 and $680 \mathrm{~m} \mu$., somewhat resembles the absorption spectrum of the plastid pigments (Fig. 16), suggesting that chlorophyll is responsible for mediating the light reaction of mating, as well as that of photosynthesis. The curve should not be interpreted too closely, however, since $(a)$ the quantum dosages employed were not equivalent for each filter, being inversely proportional to wavelength, and $(b)$ the linearity of the relation of cell-pair numbers to incident light intensities within the range of the experiment was not established.

\section{Action of inhibitors on the light reaction}

Since the action spectrum implicated chlorophyll as the sensitizing pigment, some interrelation between photosynthesis and sexual activation by light seemed probable. For this reason the influence of photosynthesis inhibitors upon these two processes was investigated. Phenylurethan, which is thought to inhibit the primary photochemical reaction, proved most useful in this

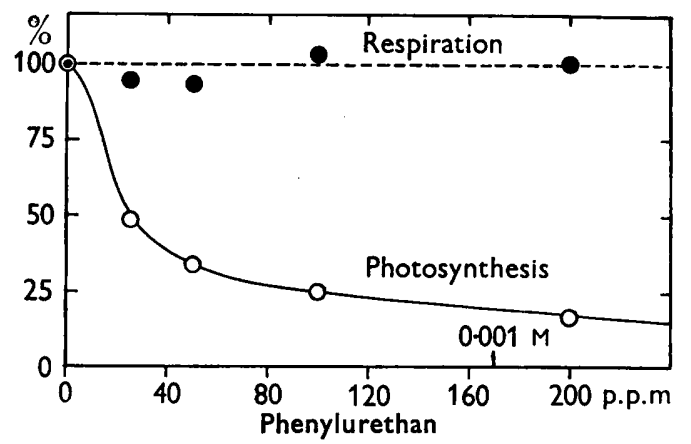

Fig. 17. Effect of phenylurethan on relative rates of respiration and photosynthesis. Plus organisms tested. Illumination 500 f.c. Temperature $25^{\circ}$. For further experimental details, see Lewin \& Mintz, 1955.

respect (Lewin \& Mintz, 1955). In certain concentrations of this narcotic (e.g. $6 \times 10^{-4} \mathrm{M}$ ) the organisms remained viable and motile, exhibiting normal or slightly augmented respiration, whereas photosynthesis was markedly inhibited (Fig. 17). It was found that this concentration of phenylurethan did not severely inhibit mating of gametes which had already been sexually activated by light; whereas gametes which had been incubated in darkness regained little sexual activity when illuminated in the presence of this narcotic (Fig. 18). No other agent among reported inhibitors of photosynthesis (see Lewin \& Mintz, 1955) was found to exhibit a similar differential inhibition. When the phenylurethan was removed by washing, the organisms regained both their power of photosynthesis and their ability to become sexually 


\section{Light and sexuality in Chlamydomonas sp.}

reactivated by light. The parallel effects of phenylurethan, on photosynthesis and on sexual reactivation, offer further support for the hypothesis that these processes have some photochemical reaction in common.



Fig. 18. Effect of phenylurethan on sexual activation in light. Darkened plus and minus suspensions mixed. Illumination 300 f.c., $60 \mathrm{~min}$. Temperature $23^{\circ}$. Pairs $/ 0 \cdot 3 \mu$ l. Direct effect of inhibitor on mating was determined by using activated organisms, which had received $60 \mathrm{~min}$. pre-illumination, in a parallel series.

\section{DISCUSSION}

The environmental factors governing the formation of gametes in Chlamydomonas moerousii closely parallel those which control the production of 'dark cells' in Chlorella ellipsoidea (Tamiya, Iwamura, Shibata, Hase \& Nihei, 1953); and it seems likely that these are homologous stages in the two genera, although sexual reproduction has not been reported in Chlorella.

The experiments reported in this paper were carried out using the number of pairs as a measure of sexual activity. Recently, Förster \& Wiese $(1954 a, b)$, using Chlamydomonas eugametos, demonstrated that cell-free filtrates from suspensions of active gametes of one mating-type may induce a clumping reaction among organisms of the complementary type. This kind of response indicates the presence of a sex-substance of type 2 (Lewin, 1954). It might be well to point out that, in the methods employed and in the activity demonstrated, the work of Förster \& Wiese has followed different lines from those reported here.

It is convenient, in discussing the effects of light on Chlamydomonas gametes, to postulate that mating activity is controlled by a hormone, a chemical agent which is elaborated in one part of the organism, presumably at the chloroplast, and which affects activity at another site, the flagellum (Lewin, 1954). There is some evidence that this hormonal agent is not liberated into the medium, since all attempts to induce sexual activity in gametes incubated in darkness, by adding cell-free filtrates from illuminated organisms, were unsuccessful. Förster \& Wiese (1954b) discussed the technical difficulties inherent in experiments attempting to demonstrate the effect of such a soluble agent (referred to by them as a B-type gamone, and by Lewin (1954) as sex-substance activity of type 3). Likewise it was not found possible to delay the loss of activity in darkness, or to induce mating activity of darkened organisms, by the passage of 
oxygen, or by the addition of chemical agents, tested over a wide range of concentrations; these included adenosine triphosphate, phosphoglycerate, diverse sugars and sugar phosphates, glutathione, cysteine, and various organic acids such as acetate and succinate.

The ability of the organisms to pair seems to fall-off in darkness at an exponential rate, an approximately linear relationship being found in any one experiment between time and the logarithm of the number of gamete pairs. This would indicate that the loss of mating activity in each organism might be attributed to a single event, such as the decay of a particle, with a fixed

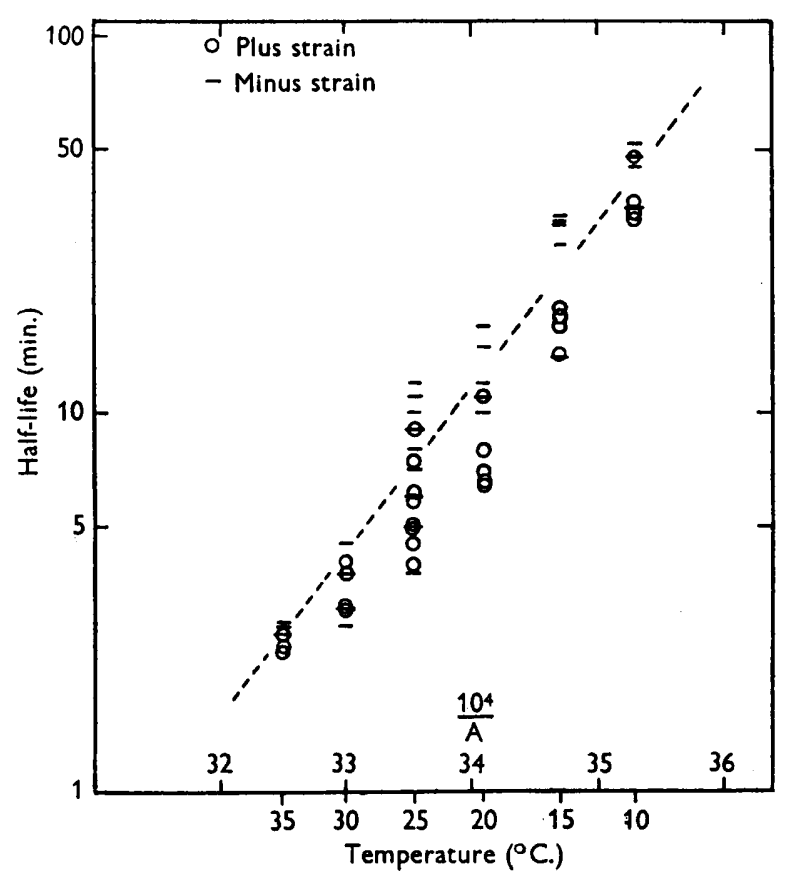

Fig. 19. Half-life values for decay of sexual activity in darkness. Each point represents a separate experiment, the slope of the decay curve being estimated by eye as in Fig. 4 .

probability of occurrence. A system of this sort is comparable with that of molecules in a first-order reaction, which react at a rate proportional to their concentration at any moment. From a plot of the logarithms of the half-life values (Table 1) against the reciprocals of the absolute temperatures (Fig. 19), the activation energy for this event may be estimated to lie in the region of 20 Kcal. per particle. (The Arrhenius theory on which this is based is discussed by Johnson, Eyring \& Polissar, 1954). This value is of doubtful significance, however, since such a one-particle mechanism appears unlikely.

It would seem more reasonable to assume that the sexual activity depends on a hormone concentration which exceeds a certain threshold, and which may vary somewhat according to the age and physical condition of each organism. If the values of concentration of hormone/organism are distributed about a mean, then as the mean value falls the number of active gametes is 
diminished. In the presence of an excess of potential mates, the number of pairs formed can be taken as a measure of the number of active gametes in which the hormone level exceeds this threshold.

These arguments are based on the hypothesis that at any one time the organisms in a suspension can be classified as sexually active or inactive. A further possibility should be considered, namely, that the individual organisms in a suspension may be more or less active. We may suggest, for instance, that in active suspensions a considerable fraction of the length of each flagellum is 'sticky', so that a high proportion of contacts between plus and minus flagella result in adhesion and ultimately in pairing. A decrease of sexual activity may then result from a decrease in the number of adhesive sites on the flagella, perhaps by the dissolution of some adhesive agent into the medium, and a consequent decrease in the frequency of fruitful contacts. Alternatively, this frequency may be controlled in some way by the concentration of a hormone within each organism. The present experimental data are not adequate to test the relative validities of these two hypotheses-whether sexual activity is an all-or-none phenomenon or not.

The fact that in darkness sexual activity is lost more rapidly under anaerobic conditions than in the presence of oxygen (Fig. 4) can be explained tentatively in at least two ways: (a) The inactivation of the hormone is a fermentative process, perhaps tied up with dark fermentations such as those reported in this species by Frenkel (1952). (b) Hormone formation is an endergonic process which proceeds rapidly in light, but which is capable of continuing slowly even in darkness, driven by the energy of endogenous respiration. However, since the rate of decay far exceeds that of synthesis, there is a net loss of mating activity in darkness, the rate of which is accelerated when respiration is arrested in anaerobiosis.

The decay of mating activity is delayed in organisms which have received some hours of illumination before being placed in darkness. This 'dark lag' period also may be explained in two ways. (a) We may consider that maximum pairing is achieved when the amount of hormone in all potential gametes reaches or exceeds the threshold level. Further illumination produces an excess, which is stored; and when such organisms are placed in darkness they retain full mating activity until the reserve of hormone has become depleted to a level below the threshold value. (b) We may suppose that mating is controlled by a photolabile inhibitor, which is accumulated when organisms are incubated in darkness. In this case, one would expect prolonged incubation to lead to the formation of excess inhibitor, which would presumably extend the lag period of the photoactivation curve. The fact that the 'light lag' appears to be unaffected by the period of prior incubation in darkness (Fig. 11) argues against this possibility.

It will be noted that, whereas the half-life values obtained for plus and minus strains are closely comparable, the duration of the dark lag tends to be considerably longer in minus than in plus suspensions under similar conditions. Förster \& Wiese $(1954 a, b)$ indicated somewhat similar behaviour in the female strain of Chlamydomonas eugametos, which is sexually equivalent to, if not 
identical with, the minus strain of $C$. moerousii (see Lewin, 1954). This difference in behaviour between the two mating-types may be taken to indicate that, in a given time, minus gametes are capable of accumulating mating hormone more rapidly than are plus gametes. Correlated with this, perhaps, are the observations that the light threshold for photoactivation at $25^{\circ}$ is lower in minus than in plus organisms, and that at 300 f.c. photoactivation occurs over a wider temperature range in minus organisms than in plus. These differences are not necessarily intrinsically bound up with mating-type differentiation, however. The two strains of $C$. moewusii may fortuitously possess many minor genetic dissimilarities, and further study would be needed to reveal whether any such difference is sex-linked or not.

The evidence from the action spectrum and from the effects of phenylurethan certainly points to some close interrelation between sexual activation and photosynthesis. A comparison between the intensities required for lightsaturation of the two processes would provide further evidence on this point. A clue to the factor controlling sexual activity was sought in the work of Calvin et al. and of Fager et al. (reviewed by Gaffron, Fager \& Rosenberg, 1951). These workers, in the course of studies on photosynthesis using radioactive ${ }^{14} \mathrm{CO}_{2}$, adduced evidence for the existence of an unstable $\mathrm{CO}_{2}$-acceptor, accumulated by Scenedesmus cells during a period of pre-illumination, and rapidly decaying in darkness. C. Ouellet \& M. Lefrançois (personal communication), using similar techniques, have calculated the half-life of the $\mathrm{CO}_{2}$ acceptor in Chlamydomonas moewusii (minus strain) to be $c .1 .5 \mathrm{~min}$. at $20^{\circ}$. At this temperature, the half-life for the loss of mating activity in darkness is about $15 \mathrm{~min}$., indicating that the two processes are unrelated. Moreover, there is no evidence that after prolonged illumination the $\mathrm{CO}_{2}$-acceptor exhibits any such lag period, prior to the onset of decay, as is exhibited in the case of mating activity. Finally, it has been shown that mating activity can be restored to inactive organisms by illumination in the absence of $\mathrm{CO}_{2}$ (other than that metabolically produced in situ), which would seem to preclude the possibility that sexual activity is controlled by the accumulation of a photosynthesized product.

This, however, does appear to be the case in certain other species of Chlamydomonas. Sager \& Granick (1954) showed that C. reinhardi organisms may be sexually active in light or darkness provided they are suspended in a medium deficient in assimilable nitrogen. Sexual activity is lost in nitrogenous media; but it may be regained when the organisms are illuminated and when, as a consequence of photosynthesis and assimilation, the medium becomes depleted of nitrogen sources. Tsubo (1956) has obtained similar results with another species, provisionally designated as Chlamydomonas sp. 24. In C. moerousii, on the other hand, sexual activity is restored by light, and maintained, in media containing ammonium or nitrate salts (0.03 $\mathrm{M} \mathrm{NaNO}_{3}, \mathrm{NH}_{4} \mathrm{Cl}, \mathrm{NH}_{4} \mathrm{NO}_{3}$ or $\left.\mathrm{Ca}\left(\mathrm{NO}_{3}\right)_{2}\right)$ just as well as in $\mathrm{N}$-free buffer (FM). It is to be hoped that the 400-odd known species of Chlamydomonas have not all evolved different mechanisms in which sexual activity may be controlled by light, and that further work will help to bring the several approaches discussed above into a more unified picture. 

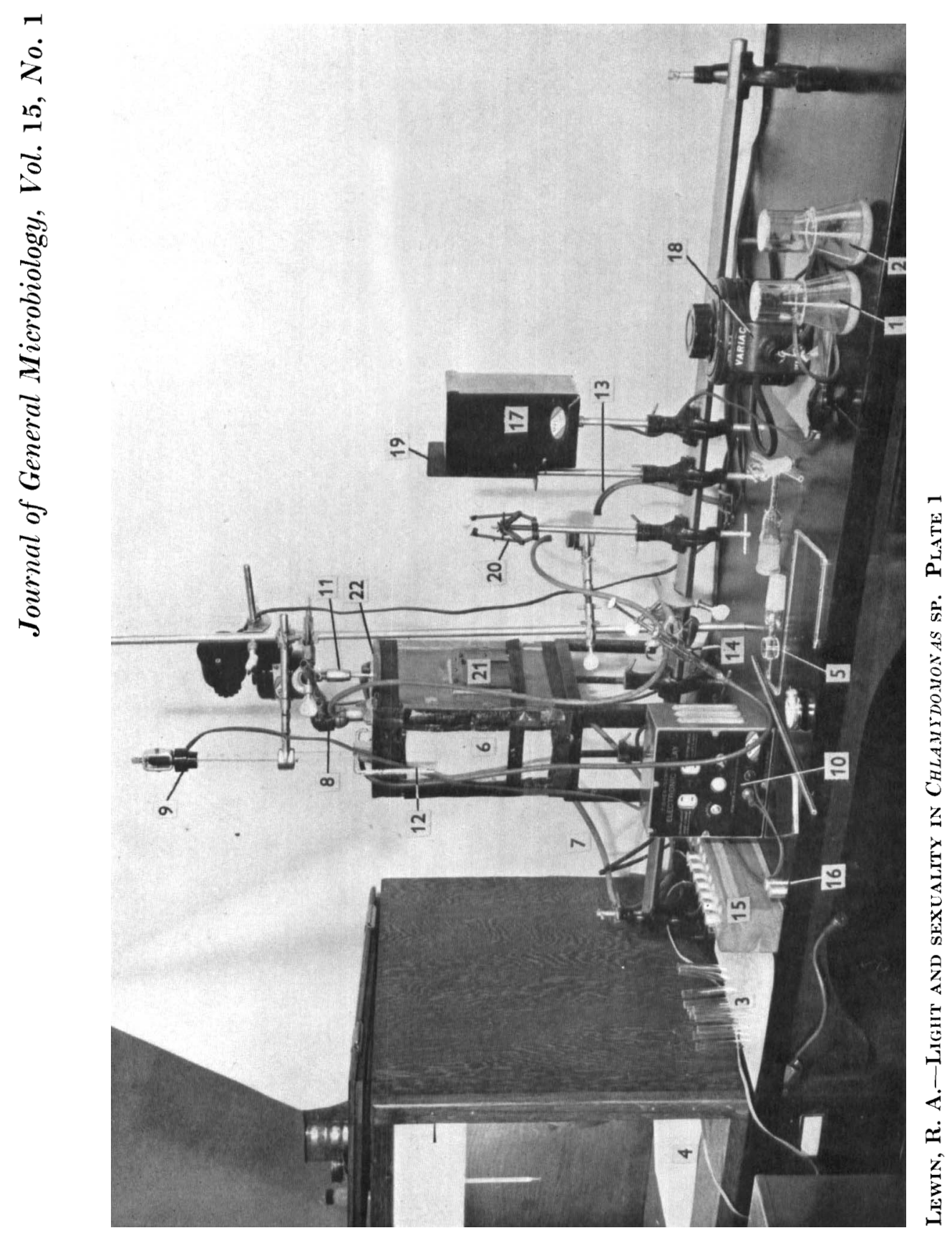

(Facing p. 185) 


\section{Light and sexuality in Chlamydomonas sp.}

Among the many colleagues and friends who have offered valuable suggestions in the course of this work and in the preparation of this paper, Dr C. Woese of Yale University and Dr C. R. Masson of the N.R.C. Laboratory in Halifax have been especially helpful and stimulating. Miss B. J. Turner and Mrs J. Menzies have assisted in the tedious task of pair-counting, employing reaction chambers constructed by Mr G. Ensell of N.R.C. Ottawa. The earlier phases of this study were carried out at Yale with the financial assistance of a Seissal Post-doctoral Fellowship. The bulk of the work was effected at the Maritime Regional Laboratory of the National Research Council of Canada. This paper is issued as N.R.C. No. 4003.

\section{REFERENCES}

FörSTER, H. \& Wiese, L. (1954a). Untersuchungen zur Kopulations-fähigkeit von Chlamydomonas eugametos. Z. Naturf. $9 b, 470$.

Förster, H. \& WiESE, L. (1954b). Gamonwirkung bei Chlamydomonas eugametos. Z. Naturf. $9 b, 548$.

Frenkel, A. W. (1952). Hydrogen evolution by the flagellate green alga, Chlamydomonas moezousii. Arch. Biochem. Biophys. 38, 219.

Gaffron, H., FAGEr, E. W. \& Rosenberg, J. L. (1951). Intermediates in photosynthesis: formation and transformation of phosphoglyceric acid. Symp. Soc. exp. Biol. 5, 262.

Johnson, F. H., Eyring, H. \& Polissar, M. J. (1954). The Kinetic Basis of Molecular Biology. New York: Wiley and Sons.

Lewin, R. A. (1953a). Studies on the flagella of algae. II. Formation of flagella by Chlamydomonas in light and darkness. Ann. N.Y. Acad. Sci. 56, 1091.

Lewin, R. A. (1953b). The genetics of Chlamydomonas moewusii Gerloff. J. Genet. 51, 543.

Lewin, R. A. (1954). Sex in unicellular algae. In Sex in Microorganisms, p. 100. Washington, D.C.: American Association for the Advancement of Science.

Lewin, R. A. \& Mintz, R. H. (1955). Inhibitors of photosynthesis in Chlamydomonas. Arch. Biochem. Biophys. 54, 246.

SAGER, R. \& Granick, S. (1954). Nutritional control of sexuality in Chlamydomonas reinhardi. J. gen. Physiol. 37, 729.

Tamiya, H., Iwamura, T., Shibata, K., Hase, E. \& Nihei, T. (1953). Correlation between photosynthesis and light-independent metabolism in the growth of Chlorella. Biochim. biophys. Acta, 12, 23.

Tsubo, Y. (1956). Observations on sexual reproduction in a Chlamydomonas. Bot. Mag., Tokyo, 69, 1.

\section{EXPLANATION OF PLATE}

Photograph of apparatus employed. Organisms are grown in flasks (1, 2). Samples of 'tester' suspension are maintained in tubes in a perspex holder (3) under constant illumination (4). A reaction chamber, similar to that shown dismantled (5), is enclosed in the constant-temperature tank (6). The water in this is cooled by running water (7) from a refrigerated tank (not shown), heated by an immersion heater (8) connected with a thermostat (9) and relay (10), and equipped with a stirrer (11). Organisms to be tested are drawn into the chamber through the capillary (12) by suction from an aspirator (13). At intervals, samples are expelled through the delivery capillary (12) by pressure on the syringe (14), and are collected in a tube of tester suspension. After being shaken, the mixture is at once darkened in the opaque wooden holder (15) and covered by an aluminium cap (16). When required, illumination of the chamber is provided by an incandescent lamp (17) connected to a rheostat (18). Light passes through an iris diaphragm (19) and is focused by a lens (20) through the perspex window (21) in the tank. Otherwise, light can be completely excluded from the interior of the tank by the light-tight lid (22) and an opaque panel covering the window (21). (Photograph F. Mason.) 